\title{
EXISTENCE AND UNIQUENESS SOLUTION FOR THREE-POINT HADAMARD-TYPE FRACTIONAL VOLTERRA BVP
}

\author{
Faraj Y. Ishak ${ }^{1 \S}$ \\ ${ }^{1}$ Department of Statistics, University of Duhok, Iraq [Email: faraj.ishak@uod.ac] \\ ${ }^{s}$ Corresponding Author
}

\begin{abstract}
This article investigates the existence and unique solution of a fractional Volterra boundary value problem of the first sort with Hadamard type and three-point boundary conditions. Our analysis is based on the fixed-point theorem of Krasnoselskii-Zabreiko and the Banach contraction principle. We explored the solution of a Hadamard type boundary value issue with fractional integral boundary conditions, and our conclusions are well demonstrated with examples.
\end{abstract}

Keywords: existence and uniqueness, fractional Volterra equation, Krasnoselskii-Zabreiko's theorem, Banach contraction principle.

\section{INTRODUCTION}

The theory of fractional differential equations and inclusions has received a lot of attention in recent years. It has become an important academic issue because to its numerous applications in the fields of physics, economics, and engineering sciences. Fractional differential equations and inclusions provide appropriate models for addressing real-world situations that cannot be addressed using classical integer-order differential equations (Benchohra et al., 2009; Ahmad et al., 2021; Bai, 2010; Balachandran \& Trujillo, 2010; Agarwal et al., 2010; Ahmad, 2010).

Fractional calculus is a branch of mathematics concerned with the study and application of arbitrary order integrals and derivatives. Fractional differential equations are derived from the mathematical modelling of systems and operations encountered in a wide variety of engineering and scientific disciplines, including physics, chemistry, aerodynamics, electrodynamics of complex media, polymer rheology, economics, control theory, signal and image processing, biophysics, and blood flow phenomena, among others (Ishak, 2020; Kilbas \& Trujillo, 2003; Guotao et al., 2018; Ahmad et al., 2021; Sial et al., 2021; Ntouyas et al., 2021; Jhanthanam et al, 2019).
The majority of study on this issue has long been recognized to be based on RiemannLiouville and Caputo-type fractional differential equations. Another type of fractional derivative that appears in the literature alongside RiemannLiouville and Caputo derivatives is the Hadamard fractional derivative introduced in 1892 (Chen et al., 2013), which is distinguished from the preceding ones by the presence of a logarithmic function of any exponent in the kernel of the integral. Details and properties of Hadamard fractional derivative and integral can be found in Ahmad et al. (2021); Samadi \& Ntouyas (2021); Kiataramkul et al. (2021); Benkerrouche et al. (2021).

This study investigated the existence and uniqueness of the following boundary value problem for the Volterra fractional differential equation of the Hadamard type.

$$
\begin{gathered}
{ }_{H} D^{\alpha} x(t)=\int_{-\infty}^{t} K(t, s) \phi(s, x(s)) d s \\
, t \in[1, w], 1<\alpha \leq 2, w \in \mathbb{R} \\
x(1)=0, \quad x(w)=\beta x(\eta), 1<\eta<w
\end{gathered}
$$

Where ${ }_{H} D^{\alpha}$ is the Hadamard derivative of order $\alpha, \phi:[1, \mathrm{w}] \times \mathbb{R} \rightarrow \mathbb{R}$ is a continuous function and $\beta$ is a real number. 


\section{PRELIMINARIES}

Definition 2.1. (Ahmad et al., 2017) The Hadamard derivative of fractional order $\mathrm{q}$ for a function $g:[1, \infty) \rightarrow \mathbb{R}$, is defined as:

$$
\begin{gathered}
{ }_{H} D^{q} g(t)=\frac{1}{\Gamma(n-q)}\left(t \frac{d}{d t}\right)^{n} \int_{1}^{t}\left(\log _{s}^{t}\right)^{n-q-1} \frac{g(s)}{s} d s \\
n-1<q<n
\end{gathered}
$$

provided the integral exists, where $[q]$ denotes the integer part of the real number $\mathrm{q}$ and $\log ()=.\log _{e}($.$) .$

Definition 2.2. (Ahmad et al., 2017) The Hadamard fractional integral of order $q \in \mathbb{R}^{+}$of a function $g \in L^{p}[a, b], 0 \leq a \leq t \leq b<\infty$ is defined as

$$
{ }_{H} I^{q} g(t)=\frac{1}{\Gamma(q)} \int_{1}^{t}\left(\log \frac{t}{s}\right)^{q-1} \frac{g(s)}{s} d s, q>0
$$

Definition 2.3. (Ahmad et al., 2017)

Let $0<a<b<\infty, \delta=t \frac{d}{d t}$ and $A C_{\delta}^{n}[a, b]=$ $\left\{f:[a, b] \rightarrow \mathbb{R}: \delta^{n-1}[f(t) \in[a, b]\}\right.$. The

Hadamard derivative of fraction order $\mathrm{q}$ for a function $f \in A C_{\delta}^{n}[a, b]$ is defined as

$$
\begin{gathered}
D^{q} f(t)=\delta^{n}\left(I^{n-q}\right)(t)= \\
\frac{1}{\Gamma(n-q)}\left(t \frac{d}{d t}\right)^{n} \int_{a}^{t}\left(\log \frac{t}{s}\right)^{n-q-1} \frac{f(s)}{s} d s
\end{gathered}
$$

Where $n-1<q<n, n=[q]+1,[q]$ denotes the integer part of the real number $q$ and $\log ()=$. $\log _{e}($.$) .$

Lemma 1. (Ahmad et al., 2017)

Let $\mathrm{q}>0$ and $x \in C(1, \infty) \cap L^{1}(1, \infty)$. Then the solution of Hadamard fractional differential equation ${ }_{H} D^{q} x(t)=0$ is given by

$$
x(t)=\sum_{i=1}^{n} c_{i}(\log t)^{q-i}
$$

And the following formula holds:

$$
{ }_{H} I_{H} D^{q} x(t)=x(t)+\sum_{i=1}^{n} c_{i}(\log t)^{q-i}
$$

where $c_{i} \in \mathbb{R}, i=1,2, \ldots, n$ and $n-1<q<$ $n$.

Rewrite problem (1) as a fixed-point problem:
${ }_{H} D^{\alpha} x(t)=\sigma(t) \quad, t \in[1, w], 1<\alpha \leq 2, w \in \mathbb{R}$

$x(1)=0, \quad x(w)=\beta x(\eta), \eta \in[1, w]$

Where $\sigma(t)=\int_{-\infty}^{t} K(t, s) \phi(s, x(s)) d s$

Lemma 2: For $1<\alpha \leq 2$ and $\sigma(\mathrm{t}) \in$ $\mathrm{C}([1, \mathrm{w}], \mathbb{R})$ the boundary value problem $(2)$ is equivalent to the integral equation:

$$
\begin{aligned}
& x(t)=\frac{1}{\Gamma(\alpha)} \int_{1}^{t}\left(\log \frac{t}{s}\right)^{\alpha-1} \frac{\sigma(s)}{s} d s+ \\
& \frac{(\log t)^{\alpha-1}}{(\log w)^{\alpha-1}-\beta(\log \eta)^{\alpha-1}}\left[\frac{\beta}{\Gamma(\alpha)} \int_{1}^{\eta}\left(\log \frac{\eta}{s}\right)^{\alpha-1} \frac{\sigma(s)}{s} d s-\right. \\
& \left.\frac{1}{\Gamma(\alpha)} \int_{1}^{w}\left(\log \frac{w}{s}\right)^{\alpha-1} \frac{\sigma(s)}{s} d s\right]
\end{aligned}
$$

Proof: In view of lemma (1) the fractional differential equation (2) is equivalent to the integral equation:

$$
\begin{aligned}
x(t)=\frac{1}{\Gamma(\alpha)} \int_{1}^{t} & \left(\log \frac{t}{s}\right)^{\alpha-1} \frac{\sigma(s)}{s} d s \\
& +c_{1}(\log t)^{\alpha-1} \\
& +c_{2}(\log t)^{\alpha-2}
\end{aligned}
$$

Using the given boundary conditions, we find that $\mathrm{c}_{2}=0$, and

$$
\begin{aligned}
& c_{1}= \\
& \frac{1}{(\log w)^{\alpha-1}-\beta(\log \eta)^{\alpha-1}}\left[\frac{\beta}{\Gamma(\alpha)} \int_{1}^{\eta}\left(\log \frac{\eta}{s}\right)^{\alpha-1} \frac{\sigma(s)}{s} d s-\right. \\
& \left.\frac{1}{\Gamma(\alpha)} \int_{1}^{w}\left(\log \frac{w}{s}\right)^{\alpha-1} \frac{\sigma(s)}{s} d s\right]
\end{aligned}
$$

Substituting the values of $c_{1}$ and $c_{2}$ in (4), we obtain (3). This completes the proof.

Now we recall the Krasnol'sk'ii-Zabreiko's fixed point theorem.

Theorem 1. (Ahmad et al., 2017)

Let $(E, \|$. $\|)$ be a Banach space, and $T: E \rightarrow E$ be a completely continuous operator. Assume that $A: E \rightarrow E$ is a bounded linear operator such that 1 is not an eigenvalue of $A$ and

$$
\lim _{\|u\| \rightarrow \infty} \frac{\|T u-A u\|}{\|u\|}=0
$$

Then $T$ has a fixed point in $E$. 
Using Lemma 2, the solution of the problem (1) can be written as:

$$
\begin{aligned}
& x(t)= \\
& \frac{1}{\Gamma(\alpha)} \int_{1}^{t}\left(\log \frac{t}{s}\right)^{\alpha-1} \frac{1}{s}\left(\int_{-\infty}^{t} K(t, s) \phi(s, x(s)) d s\right) d s+ \\
& \frac{(\log t)^{\alpha-1}}{(\log w)^{\alpha-1}-\beta(\log \eta)^{\alpha-1}} \\
& {\left[\frac{\beta}{\Gamma(\alpha)} \int_{1}^{\eta}\left(\log \frac{\eta}{s}\right)^{\alpha-1} \frac{1}{s}\left(\int_{-\infty}^{\eta} K(\eta, s) \phi(s, x(s)) d s\right) d s-\right.} \\
& \left.\frac{1}{\Gamma(\alpha)} \int_{1}^{w}\left(\log \frac{w}{s}\right)^{\alpha-1} \frac{1}{s}\left(\int_{-\infty}^{w} K(w, s) \phi(s, x(s)) d s\right) d s\right]
\end{aligned}
$$

\section{MAIN RESULT}

Consider the Banach space $X=C([1, w], \mathbb{R})$ with the norm $\|x\|=\sup _{t \in[1, w]}|x(t)|$

Theorem 2: Let $\phi$ be a continuous function, satisfying $\quad|K(t, s)|<$ $\delta e^{-\lambda(t-s)}$ for some $\delta, \lambda \in \mathbb{R}, \phi(a, 0) \neq 0$ for some $a \in[1, w]$

and

$$
\begin{aligned}
\lim _{|x| \rightarrow \infty} \frac{\phi(t, x(t))}{x} & =\Omega(t), \quad \Omega_{\max } \\
& =\max _{t \in[1, w]}|\Omega(t)|<\frac{1}{\Lambda}
\end{aligned}
$$

With

$$
\Lambda=\frac{\delta(\log w)^{\alpha}}{\lambda \Gamma(\alpha+1)}\left(1+\frac{\beta(\log \eta)^{\alpha}(\log w)^{-1}-(\log w)^{\alpha-1}}{(\log w)^{\alpha-1}-\beta(\log \eta)^{\alpha-1}}\right)
$$

Then the BVP (1) has at least one nontrivial solution in $[1, w]$.

Proof: define an operator $\Psi: X \rightarrow X$ by

$$
\begin{gathered}
\Psi x(t)=\frac{1}{\Gamma(\alpha)} \int_{1}^{t}\left(\log \frac{t}{s}\right)^{\alpha-1} \frac{\sigma(s)}{s} d s+ \\
\frac{(\log \mathrm{t})^{\alpha-1}}{(\log \mathrm{w})^{\alpha-1}-\beta(\log \eta)^{\alpha-1}}\left[\frac{\beta}{\Gamma(\alpha)} \int_{1}^{\eta}\left(\log \frac{\eta}{\mathrm{s}}\right)^{\alpha-1} \frac{\sigma(\mathrm{s})}{\mathrm{s}} \mathrm{ds}-\right. \\
\left.\frac{1}{\Gamma(\alpha)} \int_{1}^{\mathrm{w}}\left(\log \frac{\mathrm{w}}{\mathrm{s}}\right)^{\alpha-1} \frac{\sigma(\mathrm{s})}{\mathrm{s}} \mathrm{ds}\right], \mathrm{t} \in[1, \mathrm{w}]
\end{gathered}
$$

basing on this the mapping $\Psi$ is well defined, now we have to proof that there exist a fixed points for the operator $\Psi$ in the Banach space $X$. We split the proof into three steps.

Step 1. To prove that $\Psi$ is continuous let us consider a sequence $\left\{x_{n}\right\}$ converging to $x$, for each $t \in[1, w]$ we have:

$$
\begin{aligned}
& \left|\Psi x_{n}(t)-\Psi x(t)\right| \\
& \leq \frac{1}{\Gamma(\alpha)} \int_{1}^{t}\left(\log \frac{t}{s}\right)^{\alpha-1} \frac{1}{s}\left(\int_{-\infty}^{t} \mid K(t, s) \| \phi\left(s, x_{n}(s)\right)\right. \\
& -\phi(s, x(s)) \mid d s) d s+ \\
& \frac{(\log t)^{\alpha-1}}{(\log w)^{\alpha-1}-\beta(\log \eta)^{\alpha-1}} \\
& {\left[\frac { \beta } { \Gamma ( \alpha ) } \int _ { 1 } ^ { \eta } ( \operatorname { l o g } \frac { \eta } { s } ) ^ { \alpha - 1 } \frac { 1 } { s } \left(\int_{-\infty}^{\eta} K(\eta, s) \mid \phi\left(s, x_{n}(s)\right)-\right.\right.} \\
& \phi(s, x(s)) \mid d s) d s- \\
& \frac{1}{\Gamma(\alpha)} \int_{1}^{w}\left(\log \frac{w}{s}\right)^{\alpha-1} \frac{1}{s}\left(\int_{-\infty}^{w} K(w, s) \mid \phi\left(s, x_{n}(s)\right)-\right. \\
& \phi(s, x(s)) \mid d s) d s] \\
& \leq \frac{\delta\left\|\phi\left(t, x_{n}(t)\right)-\phi(t, x(t))\right\|}{\lambda \Gamma(\alpha)} \int_{1}^{t}\left(\log \frac{t}{s}\right)^{\alpha-1} \frac{1}{s} d s+ \\
& \frac{\delta(\log t)^{\alpha-1}\left\|\phi\left(t, x_{n}(t)\right)-\phi(t, x(t))\right\|}{\lambda\left((\log w)^{\alpha-1}-\beta(\log \eta)^{\alpha-1}\right)}\left[\frac{\beta}{\Gamma(\alpha)} \int_{1}^{\eta}\left(\log \frac{\eta}{s}\right)^{\alpha-1} \frac{1}{s} d s-\right. \\
& \left.\frac{1}{\Gamma(\alpha)} \int_{1}^{w}\left(\log \frac{w}{s}\right)^{\alpha-1} \frac{1}{s} d s\right] \\
& \leq \frac{\delta(\log w)^{\alpha}\left\|\phi\left(t, x_{n}(t)\right)-\phi(t, x(t))\right\|}{\lambda \Gamma(\alpha+1)} \\
& +\frac{\delta \beta(\log \mathrm{t})^{\alpha-1}(\log \eta)^{\alpha}\left\|\phi\left(t, x_{n}(t)\right)-\phi(t, x(t))\right\|}{\lambda\left((\log \mathrm{w})^{\alpha-1}-\beta(\log \eta)^{\alpha-1}\right) \Gamma(\alpha+1)} \\
& \frac{\delta(\log t)^{\alpha-1}(\log w)^{\alpha}\left\|\phi\left(t, x_{n}(t)\right)-\phi(t, x(t))\right\|}{\lambda\left((\log w)^{\alpha-1}-\beta(\log \eta)^{\alpha-1}\right) \Gamma(\alpha+1)} \\
& \leq \frac{\delta(\log w)^{\alpha}\left\|\phi\left(t, x_{n}(t)\right)-\phi(t, x(t))\right\|}{\lambda \Gamma(\alpha+1)}[1 \\
& \left.+\frac{\beta(\log \eta)^{\alpha}(\log w)^{-1}-(\log w)^{\alpha-1}}{(\log w)^{\alpha-1}-\beta(\log \eta)^{\alpha-1}}\right] \\
& \leq \Lambda\left\|\phi\left(t, x_{n}(t)\right)-\phi(t, x(t))\right\|
\end{aligned}
$$

Since the convergence of a sequence implies its boundedness therefore there exists a number $\mathrm{M}>0$ such that:

$$
\left\|x_{n}\right\| \leq \mathrm{M}, \quad\|x\| \leq \mathrm{M}
$$

and hence $\phi$ is uniformly continuous on the compact set $\{(t, x), t \in[1, w],\|x\|<M\}$, thus $\left|\Psi x_{\mathrm{n}}(\mathrm{t})-\Psi x(\mathrm{t})\right| \leq \epsilon, \forall \mathrm{n} \geq \mathrm{n}_{0}$, this shows that $\Psi$ is continuous.

For any $\mathrm{R}>0$, we consider the closed set $\mathrm{C}=$ $\{x \in X:\|x\| \leq \mathrm{R}\}$. 
Step2: we prove that $\Psi(C)$ is relatively compact in $X$ we set:

$$
\phi_{\max }=\max _{t \in[1, w],\|x\| \leq R}|\phi(t, x)|
$$

Then we have:

$$
\begin{aligned}
& |\Psi x(t)| \\
& \leq \frac{1}{\Gamma(\alpha)} \int_{1}^{t}\left(\log \frac{t}{s}\right)^{\alpha-1} \frac{1}{s}\left(\int_{-\infty}^{t}|K(t, s)||\phi(s, x(s))| d s\right) d s \\
& +\quad \frac{(\log t)^{\alpha-1}}{(\log w)^{\alpha-1}-\beta(\log \eta)^{\alpha-1}} \\
& {\left[\frac{\beta}{\Gamma(\alpha)} \int_{1}^{\eta}\left(\log \frac{\eta}{s}\right)^{\alpha-1} \frac{1}{s}\left(\int_{-\infty}^{\eta}|K(\eta, s)||\phi(s, x(s))| d s\right) d s-\right.} \\
& \left.\frac{1}{\Gamma(\alpha)} \int_{1}^{w}\left(\log \frac{w}{s}\right)^{\alpha-1} \frac{1}{s}\left(\int_{-\infty}^{w}|K(w, s)||\phi(s, x(s))| d s\right) d s\right] \\
& \leq \frac{\delta \phi_{\max }(\log w)^{\alpha}}{\lambda \Gamma(\alpha+1)}+ \\
& \frac{(\log t)^{\alpha-1}}{(\log w)^{\alpha-1}-\beta(\log \eta)^{\alpha-1}}\left[\frac{\beta \delta \phi_{\max }(\log \eta)^{\alpha}}{\lambda \Gamma(\alpha+1)}-\right. \\
& \left.\frac{\delta \phi_{\max (\log w)^{\alpha}}}{\lambda \Gamma(\alpha+1)}\right] \\
& \leq \frac{\delta \phi_{\max }(\log w)^{\alpha}}{\lambda \Gamma(\alpha+1)}[1+ \\
& \left.\frac{\beta(\log \eta)^{\alpha}(\log w)^{-1}-(\log w)^{\alpha-1}}{(\log w)^{\alpha-1}-\beta(\log \eta)^{\alpha-1}}\right] \leq \Lambda \phi_{\max }
\end{aligned}
$$

Thus $\|\Psi x\| \leq \Lambda \phi_{\max }$ and consequently $\Psi(\mathrm{C})$ is uniformly bounded. For $t_{1}, t_{2} \in$ $[1, w]$ with $t_{1}<t_{2}$ we have

$$
\begin{aligned}
& \left|\Psi x\left(t_{2}\right)-\Psi x\left(t_{1}\right)\right| \leq \\
& \frac{\delta \phi_{\max }}{\lambda \Gamma(\alpha)} \mid \int_{1}^{t_{1}}\left(\log \frac{t_{1}}{s}\right)^{\alpha-1} \frac{1}{s} d s- \\
& \int_{1}^{t_{2}}\left(\log \frac{t_{2}}{s}\right)^{\alpha-1} \frac{1}{s} d s \mid+ \\
& \frac{\beta \delta \phi_{\max }\left[\left(\log t_{2}\right)^{\alpha-1}-\left(\log t_{1}\right)^{\alpha-1}\right]}{\lambda \Gamma(\alpha)\left((\log w)^{\alpha-1}-\beta(\log \eta)^{\alpha-1}\right)} \mid \int_{1}^{\eta} \frac{\left(\log \frac{\eta}{s}\right)^{\alpha-1}}{s}- \\
& \frac{\left(\log \frac{\eta}{s}\right)^{\alpha-1}}{s} d s \mid- \\
& \frac{\delta \phi_{\max }\left[\left(\log t_{2}\right)^{\alpha-1}-\left(\log t_{1}\right)^{\alpha-1}\right]}{\lambda \Gamma(\alpha)\left((\log w)^{\alpha-1}-\beta(\log \eta)^{\alpha-1}\right)} \mid \int_{1}^{w} \frac{\left(\log \frac{w}{s}\right)^{\alpha-1}}{s}- \\
& \frac{\left(\log \frac{w}{s}\right)^{\alpha-1}}{s} d s \mid \\
& \leq \frac{\delta \phi_{\max }\left[\left(\log t_{2}\right)^{\alpha-1}-\left(\log t_{1}\right)^{\alpha-1}\right]}{\lambda \Gamma(\alpha+1)}
\end{aligned}
$$

As the right-hand side tends to 0 as $t_{1} \rightarrow t_{2}$, this guarantees that $\Psi(\mathrm{C})$ is equicontinuous by Arzela'-Ascoli theorem the mapping $\Psi$ is completely continuous on $X$. This completes the proof of Step 2.

Next consider the following boundary value problem

${ }_{H} D^{\alpha} x(t)=\theta(t) \quad, t \in[1, w], 1<\alpha \leq 2$, $w \in \mathbb{R}$

$x(1)=0, x(w)=\beta x(\eta), \eta \in$ $[1, \mathrm{w}]$

Where $\theta(t)=\int_{-\infty}^{t} K(t, s) \Omega(t) x(t) d s$.Let us define an operator $A: X \rightarrow X$ by

$\mathrm{A} x(t)=$

$$
\begin{aligned}
& \frac{1}{\Gamma(\alpha)} \int_{1}^{t}\left(\log \frac{t}{s}\right)^{\alpha-1} \frac{1}{s}\left(\int_{-\infty}^{t} K(t, s) \Omega(s) x(s) d s\right) d s+ \\
& \frac{(\log t)^{\alpha-1}}{(\log w)^{\alpha-1}-\beta(\log \eta)^{\alpha-1}} \\
& {\left[\frac{\beta}{\Gamma(\alpha)} \int_{1}^{\eta}\left(\log \frac{\eta}{s}\right)^{\alpha-1} \frac{1}{s}\left(\int_{-\infty}^{\eta} K(\eta, s) \Omega(s) x(s) d s\right) d s-\right.} \\
& \left.\frac{1}{\Gamma(\alpha)} \int_{1}^{w}\left(\log \frac{w}{s}\right)^{\alpha-1} \frac{1}{s}\left(\int_{-\infty}^{w} K(w, s) \Omega(s) x(s) d s\right) d s\right], \\
& \mathrm{t} \in[1, \mathrm{w}]
\end{aligned}
$$

Clearly, A is a bounded linear operator, in addition any fixed point of $\mathrm{A}$ is a solution of the boundary value problem (6) and vice versa.

Step 3. We now make sure that 1 is not an eigenvalue of A. Suppose that the boundary value problem (6) has a nontrivial solution $x(\mathrm{t})$, then:

$$
\begin{aligned}
& \|x\|=\|A(x)\|=\sup _{t \in[1, w]}|A x(t)| \leq \\
& \frac{1}{\Gamma(\alpha)} \int_{1}^{t}\left(\log \frac{t}{s}\right)^{\alpha-1} \frac{1}{s}\left(\int_{-\infty}^{t}|K(t, s)||\Omega(s)||x(s)| d s\right) d s+ \\
& \frac{(\log t)^{\alpha-1}}{(\log w)^{\alpha-1}-\beta(\log \eta)^{\alpha-1}} \\
& {\left[\frac{\beta}{\Gamma(\alpha)} \int_{1}^{\eta}\left(\log \frac{\eta}{s}\right)^{\alpha-1} \frac{1}{s}\left(\int_{-\infty}^{\eta}|K(\eta, s)||\Omega(s)||x(s)| d s\right) d s-\right.} \\
& \left.\frac{1}{\Gamma(\alpha)} \int_{1}^{w}\left(\log \frac{w}{s}\right)^{\alpha-1} \frac{1}{s}\left(\int_{-\infty}^{w}|K(w, s) \| \Omega(s)||x(s)| d s\right) d s\right] \\
& \leq \Omega_{m a x}\|x\|\left[\frac{\delta(\log t)^{\alpha}}{\lambda \Gamma(\alpha+1)}\right. \\
& +\frac{\delta \beta(\log t)^{\alpha-1}(\log \eta)^{\alpha}}{\lambda \Gamma(\alpha+1)\left((\log w)^{\alpha-1}-\beta(\log \eta)^{\alpha-1}\right)} \\
& \left.-\frac{\delta(\log t)^{\alpha-1}(\log w)^{\alpha}}{\lambda \Gamma(\alpha+1)\left((\log w)^{\alpha-1}-\beta(\log \eta)^{\alpha-1}\right)}\right]
\end{aligned}
$$




$$
\begin{aligned}
& \leq \Omega_{\max }\|x\| \frac{\delta(\log w)^{\alpha}}{\lambda \Gamma(\alpha+1)}[1+ \\
& \left.\frac{\beta(\log w)^{-1}(\log \eta)^{\alpha}-(\log w)^{\alpha-1}}{(\log w)^{\alpha-1}-\beta(\log \eta)^{\alpha-1}}\right] \leq \Omega_{\max } \Lambda\|x\| \leq \\
& \|x\|
\end{aligned}
$$

So, because of this contradiction the BVP (6) has no nontrivial solution. Thus 1 is not an eigenvalue of A.

Finally, we prove that:

$$
\lim _{\|x\| \rightarrow \infty} \frac{\|\Psi x-A x\|}{\|x\|}=0
$$

According to the $\lim _{|x| \rightarrow \infty} \frac{\phi(t, x(t))}{x}=\Omega(t)$, for any $\epsilon>0$ there exist some $\xi>0$ such that:

$$
|\phi(t, x(t))-\Omega(t) x|<\epsilon|x|, \text { for }|x|>\xi
$$

set

$$
\xi^{*}=\max _{t \in[1, w]}\left\{\max _{|x|<\xi} \phi(t, x(t))\right\}
$$

And select $\Delta>0$ such that:

We denote

$$
\xi^{*}+\Omega_{\max } \xi<\epsilon \Delta
$$

$$
\begin{gathered}
I_{1}=\{t \in[1, w]:|x(t)| \leq \xi\}, \quad I_{2}=\{t \\
\in[1, w]:|x(t)|>\xi\}
\end{gathered}
$$

For any $x \in X$ with $\|x\|>\Delta, t \in I_{1}$ we have:

$$
\begin{gathered}
|\phi(t, x(t))-\Omega(t) x| \\
\quad \leq|\phi(t, x(t))|+\Omega_{\text {max }}|x| \\
\leq \xi^{*}+\Omega_{\max } \xi \leq \epsilon \Delta \leq \epsilon\|x\|
\end{gathered}
$$

For any $x \in X$ with $\|x\|>\Delta, t \in I_{2}$ we have

$$
|\phi(t, x(t))-\Omega(t) x| \leq \epsilon\|x\|
$$

Then for any $x \in X$ with $\|x\|>\Delta$ we have

$$
|\phi(t, x(t))-\Omega(t) x| \leq \epsilon\|x\|
$$

Then we obtain:

$$
\begin{array}{r}
\|\Psi x-A x\|=\sup _{t \in[1, w]}|(\Psi x-A x)(t)| \\
\quad \leq \sup _{t \in[1, w]} \\
{\left[\frac { 1 } { \Gamma ( \alpha ) } \int _ { 1 } ^ { t } ( \operatorname { l o g } \frac { t } { s } ) ^ { \alpha - 1 } \frac { 1 } { s } \left(\int_{-\infty}^{t}|K(t, s)| \mid \phi(s, x(s))-\right.\right.} \\
\Omega(s) x(s) \mid d s) d s+\frac{\beta(\log t)^{\alpha-1}}{\Gamma(\alpha)\left((\log w)^{\alpha-1}-\beta(\log \eta)^{\alpha-1}\right)}
\end{array}
$$

$$
\begin{aligned}
& \int_{1}^{\eta}\left(\log \frac{\eta}{s}\right)^{\alpha-1} \frac{1}{s} \int_{-\infty}^{\eta} \mid K(\eta, s) \| \phi(s, x(s))- \\
& \Omega(s) x(s) \mid d s d s-\frac{(\log t)^{\alpha-1}}{\Gamma(\alpha)\left((\log w)^{\alpha-1}-\beta(\log \eta)^{\alpha-1}\right)} \\
& \int_{1}^{w}\left(\log \frac{w}{s}\right)^{\alpha-1} \frac{1}{s}\left(\int_{-\infty}^{w} \mid K(w, s) \| \phi(s, x(s))-\right. \\
& \Omega(s) x(s) \mid d s) d s] \\
& <\frac{\delta \epsilon\|x\|(\log t)^{\alpha}}{\lambda \Gamma(\alpha+1)}+ \\
& \frac{\delta \beta\|x\|(\log \eta)^{\alpha}(\log t)^{\alpha-1}-\delta \epsilon\|x\|(\log w)^{\alpha}(\log t)^{\alpha-1}}{\lambda \Gamma(\alpha+1)\left((\log w)^{\alpha-1}-\beta(\log \eta)^{\alpha-1}\right)} \\
& <\frac{\delta \epsilon\|x\|(\log t)^{\alpha}}{\lambda \Gamma(\alpha+1)}[1+ \\
& \left.\frac{\beta(\log \eta)^{\alpha}(\log w)^{-1}-(\log w)^{\alpha-1}}{(\log w)^{\alpha-1}-\beta(\log \eta)^{\alpha-1}}\right]<\epsilon \Lambda\|x\|
\end{aligned}
$$

Which on taking the limit yields

$$
\lim _{\|x\| \rightarrow \infty} \frac{\|\Psi x-A x\|}{\|x\|}=0
$$

Consequently,Krasnol'sk'ii-Zabreiko's theorem guarantees that the boundary value problem (1) has at last one nontrivial solution, whereas $\Psi: X \rightarrow X$ is completely continuous operator and $A: X \rightarrow X$ is bounded linear operator such that 1 is not eigenvalue of $A$ and: $\lim _{\|x\| \rightarrow \infty} \frac{\|\Psi x-A x\|}{\|x\|}=$ 0

Theorem 3: let $\phi:[1, w] \times \mathbb{R} \rightarrow \mathbb{R}$ be continuous function satisfying the following condition:

There exists a constant $L>0$ such that

$$
|\phi(t, x(t))-\phi(t, y(t))| \leq L|x-y|
$$

For each $t \in[1, w]$ and $x, y \in \mathbb{R}$, if

$$
L \Lambda<1
$$

Then the Hadamard fractional BVP (1) has a unique solution on $[1, w]$.

Proof: fixing $\max _{t \in[1, w]} \phi(t, 0)=\rho<\infty$, we define $B_{r}=\{x \in X:\|x\| \leq r\}$ Where $r \geq \frac{\rho \Lambda}{1-L \Lambda}$ ,we show that the set $B_{r}$ is invariant with respect to the operator $\Psi$ that is $\Psi B_{r} \subset B_{r}$, for $x \in B_{r}$ we have:

$$
\begin{aligned}
& \|\Psi x(t)\| \leq \\
& \max _{t \in[1, w]}\left[\frac { 1 } { \Gamma ( \alpha ) } \int _ { 1 } ^ { t } ( \operatorname { l o g } \frac { t } { s } ) ^ { \alpha - 1 } \frac { 1 } { s } \left(\int_{-\infty}^{t}|K(t, s)|(\mid \phi(s, x(s))-\right.\right. \\
& \phi(s, 0)|+| \phi(s, 0) \mid) d s) d s+ \\
& \frac{\beta(\log t)^{\alpha-1}}{\Gamma(\alpha)\left((\log w)^{\alpha-1}-\beta(\log \eta)^{\alpha-1}\right)}
\end{aligned}
$$




$$
\begin{aligned}
& \int_{1}^{\eta}\left(\log \frac{\eta}{s}\right)^{\alpha-1} \frac{1}{s}\left(\int_{-\infty}^{\eta}|K(\eta, s)|(\mid \phi(s, x(s))-\right. \\
& \phi(s, 0) \mid+ \\
& |\phi(s, 0)|) d s) d s-\frac{(\log t)^{\alpha-1}}{\Gamma(\alpha)\left((\log w)^{\alpha-1}-\beta(\log \eta)^{\alpha-1}\right)} \\
& \int_{1}^{w}\left(\log \frac{w}{s}\right)^{\alpha-1} \frac{1}{s}\left(\int_{-\infty}^{w}|K(w, s)|(\mid \phi(s, x(s))-\right. \\
& \phi(s, 0)|+| \phi(s, 0) \mid) d s) d s] \\
& \leq \frac{\delta(L r+\rho)(\log w)^{\alpha}}{\lambda \Gamma(\alpha+1)}+ \\
& \frac{\delta \beta(L r+\rho)(\log w)^{\alpha-1}(\log \eta)^{\alpha}}{\lambda \Gamma(\alpha+1)\left((\log w)^{\alpha-1}-\beta(\log \eta)^{\alpha-1}\right)}- \\
& \frac{\delta(L r+\rho)(\log w)^{\alpha-1}(\log w)^{\alpha}}{\lambda \Gamma(\alpha+1)\left((\log w)^{\alpha-1}-\beta(\log \eta)^{\alpha-1}\right)} \\
& \leq \frac{\delta(L r+\rho)(\log w)^{\alpha}}{\lambda \Gamma(\alpha+1)}[1+ \\
& \left.\frac{\beta(\log w)^{-1}(\log \eta)^{\alpha}-(\log w)^{\alpha-1}}{(\log w)^{\alpha-1}-\beta(\log \eta)^{\alpha-1}}\right] \leq(L r+\rho) \Lambda \leq r
\end{aligned}
$$

Which shows that $\Psi B_{r} \subset B_{r}$.

Now let $x, y \in X$ then for $t \in[1, w]$ we have:

$$
\begin{aligned}
& \|\Psi x(t)-\Psi y(t)\| \leq \\
& \frac{1}{\Gamma(\alpha)} \int_{1}^{t}\left(\log \frac{t}{s}\right)^{\alpha-1} \frac{1}{s}\left(\int_{-\infty}^{t} \mid K(t, s) \| \phi(s, x(s))-\right. \\
& \phi(s, y(s)) \mid d s) d s+\frac{(\log t)^{\alpha-1}}{(\log w)^{\alpha-1}-\beta(\log \eta)^{\alpha-1}} \\
& {\left[\frac { \beta } { \Gamma ( \alpha ) } \int _ { 1 } ^ { \eta } ( \operatorname { l o g } \frac { \eta } { s } ) ^ { \alpha - 1 } \frac { 1 } { s } \left(\int_{-\infty}^{\eta} \mid K(\eta, s) \| \phi(s, x(s))-\right.\right.} \\
& \phi(s, y(s)) \mid d s) d s- \\
& \frac{1}{\Gamma(\alpha)} \int_{1}^{w}\left(\log \frac{w}{s}\right)^{\alpha-1} \frac{1}{s} \int_{-\infty}^{w} \mid K(w, s) \| \phi(s, x(s))- \\
& \phi(s, y(s)) \mid d s] \\
& \quad \leq \frac{\delta L(\log w)^{\alpha}\|x-y\|}{\lambda \Gamma(\alpha+1)} \\
& \quad+\frac{\delta L(\log w)^{\alpha-1}\|x-y\|\left(\beta(\log \eta)^{\alpha}-(\log w)^{\alpha}\right)}{\lambda \Gamma(\alpha+1)\left((\log w)^{\alpha-1}-\beta(\log \eta)^{\alpha-1}\right)} \\
& \leq \frac{\delta(\log w)^{\alpha}}{\lambda \Gamma(\alpha+1)}[1+ \\
& \left.\frac{(\log w)^{-1} \beta(\log \eta)^{\alpha}-(\log w)^{\alpha-1}}{(\log w)^{\alpha-1}-\beta(\log \eta)^{\alpha-1}}\right] L\|x-y\| \leq \\
& \Lambda L\|x-y\|
\end{aligned}
$$

It follows from the assumption (7) that $\Psi$ is a contraction. In consequence by Banach's fixed point theorem the operator $\Psi$ has a fixed point which corresponds to the unique solution of problem (1). This completes the proof.

\section{APPLICATION}

The method presented in previous section is applicable to a variety of boundary value problems, and we can apply it on the following Hadamard type boundary value problem with fractional integral boundary conditions given by:

$$
\begin{gathered}
{ }_{H} D^{\alpha} x(t)=\int_{-\infty}^{t} K(t, s) \phi(s, x(s)) d s \quad, 1 \\
<t<w, \quad 1<\alpha \leq 2, \\
1<w \in \mathbb{R}^{+} \\
x(1)=0, \quad x(w)=
\end{gathered}
$$

$I^{\beta} x(\eta), \quad 1<\eta<w$

where ${ }_{H} D^{\alpha}$ is the Hadamard fractional derivative of order $\alpha, I^{\beta}$ is the Hadamard fractional integral of order $\beta$ and $\phi(t, x(t)):[1, w] \times \mathbb{R} \rightarrow \mathbb{R}$ is a continuous function.

Lemma 3: For $1<\alpha \leq 2$ and $\sigma(t) \in$ $\mathrm{C}([1, \mathrm{w}], \mathbb{R})$ the unique solution of the $\mathrm{BVP}(8)$ is equivalent to the integral equation:

$$
\begin{aligned}
& x(t)=\frac{1}{\Gamma(\alpha)} \int_{1}^{t}\left(\frac{\log t}{s}\right)^{\alpha-1} \frac{\sigma(s)}{s} d s+ \\
& \frac{(\log t)^{\alpha-1}\left[\frac{1}{\Gamma(\beta+\alpha)} \int_{1}^{\eta}\left(\log \frac{\eta}{s}\right)^{\beta+\alpha-1} \frac{\sigma(s)}{s} d s-\frac{1}{\Gamma(\alpha)} \int_{1}^{w}\left(\log \frac{w}{s}\right)^{\alpha-1} \frac{\sigma(s)}{s} d s\right]}{(\log w)^{\alpha-1}-\frac{1}{\Gamma(\beta)} \int_{1}^{\eta}\left(\log \frac{\eta}{s}\right)^{\beta-1} \frac{(\log s)^{\alpha-1}}{s} d s}(9)
\end{aligned}
$$

Where $\sigma(t)=\int_{-\infty}^{t} K(t, s) \phi(s, x(s)) d s$.

Proof: In view of lemma (2) the fractional differential equation (8) is equivalent to the integral equation:

$$
\begin{aligned}
& x(t)=\frac{1}{\Gamma(\alpha)} \int_{1}^{t}\left(\log \frac{\mathrm{t}}{\mathrm{s}}\right)^{\alpha-1} \frac{\sigma(\mathrm{s})}{\mathrm{s}} \mathrm{ds}+ \\
& \mathrm{c}_{1}(\log \mathrm{t})^{\alpha-1}+\mathrm{c}_{2}(\log \mathrm{t})^{\alpha-2}
\end{aligned}
$$

Using the given boundary conditions, we find that $\mathrm{c}_{2}=0$, and

$$
c_{1}=\frac{I^{\beta+\alpha} \sigma(\eta)-I^{\alpha} \sigma(w)}{(\log w)^{\alpha-1}-\frac{1}{\Gamma(\beta)} \int_{1}^{\eta}\left(\log \frac{\eta}{s}\right)^{\beta-1} \frac{(\log s)^{\alpha-1}}{s} d s}
$$


Substituting the values of $c_{1}$ and $c_{2}$ in (10) we obtain (9). This completes the proof.

Theorem 4: Let $\varphi$ be a continuous function, satisfying $|G(t, s)|<$ $\delta_{1} e^{-\lambda_{1}(t-s)}$ for some $\delta_{1} \lambda_{1}, \in \mathbb{R}, \varphi(a, 0) \neq 0$ for some $a \in[1, w]$

and

$$
\begin{aligned}
\lim _{|x| \rightarrow \infty} \frac{\varphi(t, x(t))}{x} & =\mathrm{\varrho}(t), \quad \varrho_{\max } \\
& =\max _{t \in[1, w]}|\varrho(t)|<\frac{1}{\Lambda_{1}}
\end{aligned}
$$

With

$$
\begin{gathered}
\Lambda_{1}=\frac{\delta(\log w)^{\alpha-1}}{\lambda \Gamma(\alpha+1)}+ \\
\frac{\beta(\log \eta)^{\alpha+\beta}(\log w)^{\alpha-1}}{\lambda \Gamma(\alpha+\beta+1)}+\frac{\delta(\log w)^{2 \alpha-1}}{\lambda \Gamma(\alpha+1)} \\
\frac{(\log w)^{\alpha-1}-\frac{\Gamma(\alpha)}{\Gamma(\alpha+\beta)}(\log \eta)^{\alpha+\beta-1}}{}
\end{gathered}
$$

Then BVP (8) has at least one nontrivial solution in $[1, w]$.

Proof: define an operator $\mathfrak{T}: X \rightarrow X$ by:

$$
\begin{aligned}
& \mathfrak{S} x(t)=\frac{1}{\Gamma(\alpha)} \int_{1}^{t}\left(\frac{\log t}{s}\right)^{\alpha-1} \frac{\sigma(s)}{s} d s+ \\
& \frac{(\log t)^{\alpha-1}\left[\frac{1}{\Gamma(\beta+\alpha)} \int_{1}^{\eta}\left(\log \frac{\eta}{s}\right)^{\beta+\alpha-1} \frac{\sigma(s)}{s} d s-\frac{1}{\Gamma(\alpha)} \int_{1}^{w}\left(\log \frac{w}{s}\right)^{\alpha-1} \frac{\sigma(s)}{s} d s\right]}{(\log w)^{\alpha-1}-\frac{1}{\Gamma(\beta)} \int_{1}^{\eta}\left(\log \frac{\eta}{s}\right)^{\beta-1} \frac{\log s)^{\alpha-1}}{s} d s}
\end{aligned}
$$

,$t \in[1, w]$

We omit the further details as the remaining proof runs parallel to that of Theorem (2) with $\Lambda_{1}$ in place of $\Lambda$.

Theorem 5: let $\varphi:[1, w] \times \mathbb{R} \rightarrow \mathbb{R}$ be continuous function satisfying the following condition:

There exists a constant $L_{1}>0$ such that

$$
|\varphi(t, x(t))-\varphi(t, y(t))| \leq L_{1}|x-y|
$$

For each $t \in[1, w]$ and $x, y \in \mathbb{R}$, if

$$
L_{1} \Lambda_{1}<1
$$

Then the Hadamard fractional BVP (8) has a unique solution on $[1, \mathrm{w}]$.

The details of the proof have been omitted because they are parallel to what is found in Theorem (3)

\section{EXAMPLES}

Example 1: Consider the boundary value problem

$$
\begin{gathered}
D^{1.25} x(t)=\int_{-\infty}^{t} e^{1-2 t}((\sin t+1) x(t)+ \\
1) d s \quad, t \in[1,2] \\
x(1)=0, \quad x(2)= \\
1.5 x(1.5), \quad \eta \in[1, w]
\end{gathered}
$$

Here $\alpha=1.25, \beta=1.5, \eta=1.5, w=2$, and $K(t, s)=e^{1-2 t}<e^{-2(t-2)}$ where $\delta=1, \lambda=$ $2, \phi(t, x(t))=(\sin t+1) x(t)+1$ where $\phi(a, 0)=1 \neq 0$ and

$$
\begin{gathered}
\begin{aligned}
& \lim _{|x| \rightarrow \infty} \frac{\phi(t, x(t))}{x}=\sin t+1=\Omega(t) \rightarrow \Omega_{\max } \\
&=0.636432 \\
& \Lambda=\frac{\delta(\log w)^{\alpha}}{\lambda \Gamma(\alpha+1)}(1+ \\
&\left.\frac{\beta(\log \eta)^{\alpha}(\log w)^{-1}-(\log \mathrm{w})^{\alpha-1}}{(\log \mathrm{w})^{\alpha-1}-\beta(\log \eta)^{\alpha-1}}\right)=0.6255956
\end{aligned}
\end{gathered}
$$

Then $\Omega_{\max } \Lambda \approx 0.398149<1$, and hence by Theorem (2) the boundary value problem (11) has at least one solution.

On the other hand since $\mid \phi(t, x(t))-$ $\phi(t, y(t))|=| x(t) \sin t+x(t)-y(t) \sin t+$ $y(t)|\leq| x-y|| \sin t+1|\leq L| x-y \mid$, where $L \geq \sin t+1=1.03, t \in[1,2]$, and $L \Lambda<1$ then by theorem (3) the BVP (11) has a unique solution on $[1,2]$.

Example 2: consider the boundary value problem

$D^{1.25} x(t)=\int_{-\infty}^{t} e^{1-2 t}((\sin t+1) x(t)+1) d s$,

$$
t \in[1,2]
$$

$$
\begin{gathered}
x(1)=0, x(2)=I^{1.5} x(1.5), \\
\eta \in[1, w]
\end{gathered}
$$

Here $\alpha=1.25, \beta=1.5, \eta=1.5, w=2$, and $G(t, s)=e^{1-2 t}<e^{-2(t-2)}$ where $\delta_{1}=$ $1, \lambda_{1}=2, \quad \varphi(t, x(t))=(\sin t+1) x(t)+1$ where $\varphi(a, 0)=1 \neq 0$ and 


$$
\begin{gathered}
\lim _{|x| \rightarrow \infty} \frac{\varphi(t, x(t))}{x}=\sin t+1=\mathrm{Q}(t) \rightarrow \mathrm{\varrho}_{\max } \\
=0.636432 \\
\Lambda_{1}=\frac{\delta(\log w)^{\alpha-1}}{\lambda \Gamma(\alpha+1)}+ \\
\frac{\frac{\beta(\log \eta)^{\alpha+\beta}(\log w)^{\alpha-1}}{\lambda \Gamma(\alpha+\beta+1)}+\frac{\delta(\log w)^{2 \alpha-1}}{\lambda \Gamma(\alpha+1)}}{(\log \mathrm{w})^{\alpha-1}-\frac{\Gamma(\alpha)}{\Gamma(\alpha+\beta)}(\log \eta)^{\alpha+\beta-1}}=0.41348649
\end{gathered}
$$

Then $\Lambda \varrho_{\max } \approx 0.263156<1$, and hence by Theorem (4) the boundary value problem (12) has at least one solution.

On the other hand since $\mid \varphi(t, x(t))-$ $\varphi(t, y(t))|=| x(t) \sin t+x(t)-y(t) \sin t+$ $y(t)|\leq| x-y|| \sin t+1\left|\leq L_{1}\right| x-y \mid$, where $L_{1} \geq \sin t+1=1.03, t \in[1,2]$, and $\quad L \Lambda<1$ then by theorem (5) the BVP (12) has a unique solution on $[1,2]$.

\section{CONCLUSION}

In this research paper we have proven the existence and uniqueness of solutions for the Hadamard type Volterra fractional integrodifferential equation with three-point boundary value conditions by selecting $1<\alpha \leq$ 2 and optional interval $[1, w]$. Boundary value conditions have been chosen to contain three different point for which have never been used together with Volterra equation before in any article as far as we know. Existence of solutions have been shown by Krasnol'sk'ii-Zabreiko's fixed point theorem, and uniqueness solutions have been investigated by Banach contraction principal theorem.

The case of fractional integral boundary conditions was discussed, examples have been supported in order to demonstrate all theorems very well.

\section{REFERENCES}

Kilbas, A.A., \& Trujillo, J.J. (2003). Hadamardtype integrals as G-transforms, Integral Transform. Spec. Funct. 14, 413427. Doi: 10.1080/1065246031000074443.

Ahmad, B. (2010). Existence of solutions for fractional differential equations of order with anti-periodic boundary conditions. J. Appl. Math. Comput. 34, 385-391. doi.org/10.1007/s12190-009-0328-4.
Ahmad, B., \& Ntouyas, S.K. (2010). Some existence results for boundary value problems of fractional differential inclusions with non-separated boundary conditions, Electron. J. Qual. Theory Differ. Equ., 71, 117. doi.org/10.14232/ejqtde.2010.1.71.

Ahmad, B., \& Ntouyas, S.K. (2021). HilferHadamard Fractional Boundary Value Problems with Nonlocal Mixed Boundary Conditions. Fractal Fract., 5, 195. https://doi.org/10.3390/fractalfract5040195.

Ahmad, B., Ntouyas, S.K., Alsaedi, A. et al. (2021). A study of a coupled system of Hadamard fractional differential equations with nonlocal coupled initial-multipoint conditions. Adv Diff. Equ., 33. doi.org/10.1186/s13662-020-03198-4.

Ahmad, B., Alsaedi, A., Ntouyas, S.K., \& Jessada, T. (2017). Hadamard-type fractional differential equations, inclusions and inequalities. doi.org/10.1007/978-3319-52141-1.

Agarwal, R.P., Benchohra, M., \& Hamani, S. (2010). A survey on existence results for boundary value problems of nonlinear fractional differential equations and inclusions. Acta Appl. Math., 109, 973-1033. doi.org/10.1007/s10440-008-9356-6.

Bai, Z.B. (2010). On positive solutions of a nonlocal fractional boundary value problem. Nonlinear Anal., 72, 916-924. doi.org/10.1016/j.na.2009.07.033.

Balachandran, K, \& Trujillo, J.J. (2010). The nonlocal Cauchy problem for nonlinear fractional integrodifferential equations in Banach spaces. Nonlinear Anal., 72, 45874593. doi.org/10.1016/j.na.2010.02.035.

Benchohra, M., Hamani, S., \& Ntouyas, S.K. (2009). Boundary value problems for differential equations with fractional order and nonlocal conditions, Nonlinear Anal., 71, 2391-2396. doi.org/10.1016/j.na.2009.01.073. 
Benkerrouche, A., Souid, M.S., Etemad, S. Hakem, A. Agarwal, P., Rezapour, S., Ntouyas, S.K., \& Tariboon, J. (2021). Qualitative Study on Solutions of a Hadamard Variable Order Boundary Problem via the Ulam-Hyers-Rassias Stability. Fractal Fract., 5, 108. https://doi.org/10.3390/fractalfract5030108.

Ishak, F. Y. (2020). Existence Solution for Nonlinear System of Fractional Integrodifferential Equations of Volterra Type with Fractional Boundary Conditions. JMM, 6(1), 1-12. doi.org/10.15642/mantik.2020.6.1.1-12.

Guotao, W., Ke, P., Ravi, P., Agarwal, Lihong. Z., \& Ahmad, B. (2018). Nonlocal Hadamard fractional boundary value problem with Hadamard integral and discrete boundary conditions on a half-line, J Comput Appl Math, 343, 230-239. https://doi.org/10.1016/j.cam.2018.04.062.

Jhanthanam, S.. Tariboon, J., \& Ntouyas, S.K. (2019). Nonlaopon, K. On q-HermiteHadamard Inequalities for Differentiable Convex Functions. Mathematics, 7, 632. https://doi.org/10.3390/math7070632.

Kiataramkul, C., Yukunthorn, W., Ntouyas, S.K., \& Tariboon, J. (2021). Sequential Riemann-Liouville and Hadamard-Caputo Fractional Differential Systems with Nonlocal Coupled Fractional Integral Boundary Conditions. Axioms, 10, 174. https://doi.org/10.3390/axioms10030174
Ntouyas, S.K., Sitho, S., Khoployklang, T., \& Tariboon, J. (2021). Sequential RiemannLiouville and Hadamard-Caputo Fractional Differential Equation with Iterated Fractional Integrals Conditions. Axioms, 10, 277. https://doi.org/10.3390/axioms10040277.

Sial, I.B., Ali, M.A., Murtaza, G., Ntouyas, S.K., Soontharanon, J., \& Sitthiwirattham, T. (2021). On Some New Inequalities of Hermite-Hadamard Midpoint and Trapezoid Type for Preinvex Functions in p,q-Calculus. Symmetry. 13, 1864. https://doi.org/10.3390/sym13101864.

Samadi, A., \& Ntouyas, S. (2021). Coupled systems of Caputo-Hadamard differential equations with coupled Hadamard fractional integral boundary conditions. Acta Mathematica Universitatis Comenianae, 118. Retrieved, www.iam.fmph.un iba.sk/amuc/ojs/index.php/amuc/article/vie w/1639/897

Chen, Y. Tang, X., \& He, X. (2013). Positive solutions of fractional differential inclusions at resonance, Mediterr. J. Math. 10, 12071220. doi.org/10.1007/s00009-013-0273-1. 